\title{
Proton and hydrogen atom transport in the Martian upper atmosphere with an induced magnetic field
}

\author{
V. I. Shematovich, ${ }^{1}$ D. V. Bisikalo, ${ }^{1}$ C. Diéval, ${ }^{2}$ S. Barabash, ${ }^{2}$ G. Stenberg, ${ }^{2}$ H. Nilsson, ${ }^{2}$ \\ Y. Futaana, ${ }^{2}$ M. Holmstrom, ${ }^{2}$ and J.-C. Gérard ${ }^{3}$ \\ Received 14 July 2011; revised 9 September 2011; accepted 10 September 2011; published 17 November 2011.
}

[1] We have applied the Direct Simulation Monte Carlo method to solve the kinetic equation for the $\mathrm{H} / \mathrm{H}^{+}$transport in the upper Martian atmosphere. We calculate the upward $\mathrm{H}$ and $\mathrm{H}^{+}$fluxes, values that can be measured, and the altitude profile of the energy deposition to be used to understand the energy balance in the Martian atmosphere. The calculations of the upward flux have been made for the Martian atmosphere during solar minimum. We use an energy spectrum of the down moving protons in the altitude range 355-437 km adopted from the Mars Express Analyzer of Space Plasma and Energetic Atoms measurements in the range $700 \mathrm{eV}-20 \mathrm{keV}$. The particle and energy fluxes of the downward moving protons were equal to $3.0 \times 10^{6} \mathrm{~cm}^{-2} \mathrm{~s}^{-1}$ and $1.4 \times 10^{-2} \mathrm{erg} \mathrm{cm}^{-2} \mathrm{~s}^{-1}$. It was found that $22 \%$ of particle flux and $12 \%$ of the energy flux of the precipitating protons is backscattered by the Martian upper atmosphere, if no induced magnetic field is taken into account in the simulations. If we include a $20 \mathrm{nT}$ horizontal magnetic field, a typical field measured by Mars Global Surveyor in the altitude range of $85-500 \mathrm{~km}$, we find that up to $40 \%-50 \%$ of the energy flux of the precipitating protons is backscattered depending on the velocity distribution of the precipitating protons. We thus conclude that the induced magnetic field plays a crucial role in the transport of charged particles in the upper atmosphere of Mars and, therefore, that it determines the energy deposition of the solar wind.

Citation: Shematovich, V. I., D. V. Bisikalo, C. Diéval, S. Barabash, G. Stenberg, H. Nilsson, Y. Futaana, M. Holmstrom, and J.-C. Gérard (2011), Proton and hydrogen atom transport in the Martian upper atmosphere with an induced magnetic field,

J. Geophys. Res., 116, A11320, doi:10.1029/2011JA017007.

\section{Introduction}

[2] Mars differs from the Earth in what concerns the atom and ion precipitation onto the atmosphere. At the Earth a strong intrinsic magnetic field diverts the solar wind flow around the planet far above the neutral atmosphere and only the magnetic cusps can channel the particles and energy down to the atmosphere. Instead, on Mars the boundary between the solar wind and the obstacle, the induced magnetosphere boundary (IMB), is located so close to the planet that the solar wind particles penetrating IMB can directly interact with the upper atmosphere. These differences between the solar wind interaction at Earth and Mars result in different characteristics of the precipitating particles. The terrestrial ring current protons and heavier ions accelerated up to energies of tens $\mathrm{keV}$ in the magnetosphere are guided by the strong global intrinsic magnetic field and reach the upper atmosphere at high altitudes [Moore and Horwitz, 2007]. In

\footnotetext{
${ }^{1}$ Institute of Astronomy, Russian Academy of Sciences, Moscow, Russia.

${ }^{2}$ Swedish Institute of Space Physics, Kiruna, Sweden.

${ }^{3}$ LPAP, Université de Liège, Liège, Belgium.
}

Copyright 2011 by the American Geophysical Union. 0148-0227/11/2011JA017007 contrast, Mars has no ring current and the proton energies near the planet do not exceed a few $\mathrm{keV}$. The induced magnetic field near the planets is typically weak, only several tens of $\mathrm{nT}$. The relative importance of different collision processes between protons and atmospheric neutrals is also expected to be different at Mars and at the Earth because of the different precipitating energies and the different neutral compositions [Kallio and Barabash, 2001]. Therefore, studying the $\mathrm{H} / \mathrm{H}^{+}$ transport in the upper Martian atmosphere presents a new scientific challenge.

[3] Lundin et al. [2004] used the in situ plasma data of the Analyzer of Space Plasma and Energetic Atoms (ASPERA-3) experiment on board the Mars Express mission to show that solar wind protons can reach altitudes as low as $270 \mathrm{~km}$ at Mars. Futaana et al. [2006] reported energetic neutral atom (ENA) fluxes from the Martian dayside at low altitudes. They interpreted the ENA flux as a result of the backscattering of neutralized solar wind protons from the atmosphere and charge-exchanged neutral hydrogen of solar wind proton origin, the so-called ENA albedo [Kallio and Barabash, 2001]. The Martian upper atmosphere is also a subject of the solar wind ENAs precipitation originated from the charge exchange reaction between the solar wind protons outside the IMB and the extended hydrogen corona. The total energy flux 
associated to the precipitating $(\leq 1 \mathrm{keV})$ hydrogen atoms can be substantial $\sim 10^{9} \mathrm{eV} \mathrm{cm}^{-2} \mathrm{~s}^{-1}$ [Kallio et al., 1997]. Therefore, the atmospheric effects caused by these precipitating atoms and protons should be also investigated.

[4] To investigate the transport of protons and hydrogen ENAs through the upper Martian atmosphere we developed the Direct Simulation Monte Carlo (DSMC) model. The main features of the model are discussed in section 2. In section 3 results of simulations are presented. Section 4 summarizes the results and conclusions.

\section{The Model Description}

\subsection{Proton/Hydrogen Precipitation}

[5] Interactions of precipitating energetic hydrogen atoms and protons with the main atmospheric constituents include the momentum and energy transfer in elastic and inelastic collisions, ionization of target atmospheric molecules/atoms, charge transfer and electron capture collisions. Energetic $\mathrm{H}$ atoms (or protons) produced by proton (or hydrogen) impact further collide with the main atmosphere constituents, transferring their momentum and kinetic energy to atmospheric particles by elastic and inelastic collisions, ionization and stripping processes. The collisional processes describing the penetration of the energetic $\mathrm{H}^{+} / \mathrm{H}$ through the ambient atmospheric gas can be written as:

$$
H^{+}(H)+M \rightarrow\left\{\begin{array}{c}
H_{f^{\prime}}^{+}\left(H_{f^{\prime}}\right)+M^{*} \\
H_{f^{\prime}}^{+}\left(H_{f^{\prime}}\right)+M^{+}+e \\
H_{f^{\prime}}\left(H_{f^{\prime}}^{+}\right)+M^{+}(M)+(e) .
\end{array}\right.
$$

Here, $\mathrm{M}$ denotes the major atmospheric constituents $-\mathrm{CO}_{2}$, $\mathrm{N}_{2}$, and $\mathrm{O}$ included in the model. Secondary fast $\mathrm{H}_{\mathrm{f}^{\prime}}$ atoms and $\mathrm{H}_{\mathrm{f}^{\prime}}^{+}$protons produced by momentum transfer and stripping reactions loop the reaction set shown above. Consequently, the interaction of the precipitating hydrogen atoms (protons) with the main neutral constituents of the thermosphere must be considered as a cascade process producing a growing set of translationally and internally excited particles $\mathrm{M}^{*}$ of the ambient atmospheric gas.

\subsection{Mathematical Description}

[6] To analyze the penetration of energetic $\mathrm{H} / \mathrm{H}^{+}$into the atmospheric gas, we use the kinetic Boltzmann equations [Gérard et al., 2000] with the collision term:

$$
\begin{array}{r}
\mathbf{v} \frac{\partial}{\partial \mathbf{r}} f_{H / H+}+\left(\mathbf{g}+\frac{e}{m_{H+}} \mathbf{v} \times \mathbf{B}\right) \frac{\partial}{\partial \mathbf{v}} f_{H / H+} \\
=Q_{H / H+}(\mathbf{v})+\sum_{M=O, N_{2}, C O_{2}} J_{m t}\left(f_{H / H+}, f_{M}\right),
\end{array}
$$

where $\mathrm{f}_{H / H^{+}}(\mathbf{r}, \mathbf{v})$, and $\mathrm{f}_{\mathrm{M}}(\mathbf{r}, \mathbf{v})$ are the velocity distribution functions for hydrogen atoms or protons, and components of ambient gas, respectively, e the electron charge, and $\mathrm{m}_{\mathrm{H}+}$ the proton mass. The left side of the kinetic equation describes the transport of particles in the planetary gravitational and induced magnetic fields. The right-hand side term $\mathrm{Q}_{\mathrm{H} / \mathrm{H}^{+}}$is the production rate of respective particles in charge exchange and stripping collisions. The elastic and inelastic scattering terms $\mathrm{J}_{\mathrm{mt}}$ for $\mathrm{H} / \mathrm{H}^{+}$collisions with the ambient atmospheric species are written in the standard form [Shematovich et al., 1994]. It is assumed that the ambient atmospheric gas is characterized by local Maxwellian velocity distribution functions.

\subsection{Stochastic Approach}

[7] The DSMC method used to solve the kinetic equation (1) implies generation of a sample of paths for the state of the physical system under study $-\mathrm{H} / \mathrm{H}^{+}$thermalization and transport in the transition region of the upper atmosphere. It is an efficient tool for studying such complex kinetic systems in the stochastic approximation [Shematovich et al., 1994; Bisikalo et al., 1995; Gérard et al., 2000]. The details of the algorithmic realization of this numeric model were given earlier [Shematovich et al., 1994; Bisikalo et al., 1995]. The statistics in the DSMC model is controlled using the standard procedures [Shematovich, 2008]. When the steady state is reached then it is possible to accumulate the statistics with the needed accuracy. In the calculations presented below the fluxes and other characteristics were calculated with the variation below $10 \%$. The low-energy parts of proton and hydrogen fluxes were calculated with the sufficient accuracy because these particles were slowed down due to the wealth of collisions with the ambient atmospheric gas.

[8] The energy deposition rate of $\mathrm{H} / \mathrm{H}^{+}$flux is determined by the cross sections of the collisions with the ambient gas. The energy lost by the $\mathrm{H} / \mathrm{H}^{+}$in a collision is determined by the scattering angle $\chi$

$$
\Delta \mathbf{E}=\mathbf{E}_{H / H+} \times\left(\frac{2 m_{M} m_{H / H+}}{\left(m_{M}+m_{H / H+}\right)^{2}}\right) \times(1-\cos \chi),
$$

where $\mathrm{E}_{\mathrm{H} / \mathrm{H}+}$ is the initial energy of the impacting proton or hydrogen atom. It is apparent that the energy loss for collisions in forward direction (for $\chi<90^{\circ}$ ) at small scattering angles $\chi$ is less than that for larger scattering angles. The key point of this model is the stochastic treatment of the scattering angle distribution. This distribution influences both the energy degradation rate through the losses of energy in the momentum transfer collisions that are proportional to the sine of the scattering angle, and the angle redistribution of the precipitating protons.

\subsection{Numerical Model}

[9] We consider the $\mathrm{H} / \mathrm{H}^{+}$transport between altitudes where $\mathrm{H} / \mathrm{H}^{+}$are efficiently thermalized and atmospheric gas becomes practically collisionless. For the Martian atmosphere, the lower boundary is placed at $80 \mathrm{~km}$. The $80 \mathrm{~km}$ altitude is well below the exobase placed near $180 \mathrm{~km}$ at low solar activity conditions. To link our model with the actual measurements we chose the upper boundary in the altitude range $355-437 \mathrm{~km}$, where measurements of the precipitating protons were made by the ASPERA-3 instrument (see Table 1 for details). The region of the atmosphere under the study was divided into 49 vertical cells, and the altitude-dependent cell size is chosen according to the condition that it must be equal to or smaller than the free path length. In model the radial position and 3 velocity components for each modeling particle are kept. The modeling particle trajectory is calculated in 3-D space for each time step and after that the new radial position is kept. Therefore, protons launched at given angle 
Table 1. Input Data for the DSMC Model

\begin{tabular}{|c|c|c|c|c|}
\hline Run & B, Horizontal (nT) & $\begin{array}{c}\text { Precipitating } \mathrm{H}^{+} \text {or } \\
\text { H Distribution } \\
\text { Versus Nadir }\end{array}$ & $\begin{array}{l}\text { Precipitating } \mathrm{H}^{+} \text {Spectrum Measured } \\
\text { by ASPERA-3 at Altitude } \mathrm{h}_{\mathrm{ub}}\end{array}$ & Precipitating H Spectrum \\
\hline 1 & 0 & Isotropic, $0^{\circ}-90^{\circ}$ & Spectrum $1, \mathrm{~h}_{\mathrm{ub}}=437 \mathrm{~km}$ & None \\
\hline 2 & 10 & Isotropic, $0^{\circ}-90^{\circ}$ & Spectrum $1, \mathrm{~h}_{\mathrm{ub}}=437 \mathrm{~km}$ & None \\
\hline $3 a$ & 20 & Isotropic, $0^{\circ}-90^{\circ}$ & Spectrum $1, \mathrm{~h}_{\mathrm{ub}}=355 \mathrm{~km}$ & None \\
\hline $3 b$ & 20 & Isotropic, $0^{\circ}-90^{\circ}$ & Spectrum $1, \mathrm{~h}_{\mathrm{ub}}=437 \mathrm{~km}$ & None \\
\hline 4 & 30 & Isotropic, $0^{\circ}-90^{\circ}$ & Spectrum $1, \mathrm{~h}_{\mathrm{ub}}=437 \mathrm{~km}$ & None \\
\hline 5 & 50 & Isotropic, $0^{\circ}-90^{\circ}$ & Spectrum $1, \mathrm{~h}_{\mathrm{ub}}=437 \mathrm{~km}$ & None \\
\hline $6 a$ & 20 & Isotropic, $0^{\circ}-90^{\circ}$ & Spectrum $2, \mathrm{~h}_{\mathrm{ub}}=387 \mathrm{~km}$ & None \\
\hline $6 b$ & 20 & Isotropic, $0^{\circ}-90^{\circ}$ & Spectrum $2, \mathrm{~h}_{\mathrm{ub}}=435 \mathrm{~km}$ & None \\
\hline 7 & 20 & Isotropic, $0^{\circ}-90^{\circ}$ & None, $\mathrm{h}_{\mathrm{ub}}=500 \mathrm{~km}$ & MS H ${ }^{\mathrm{a}}[$ Kallio et al., 1997] \\
\hline 8 & 20 & Isotropic, $0^{\circ}-90^{\circ}$ & None, $\mathrm{h}_{\mathrm{ub}}=500 \mathrm{~km}$ & $\mathrm{SW} \mathrm{H}^{\mathrm{b}}[$ Kallio et al., 1997] \\
\hline
\end{tabular}

${ }^{a}$ Hydrogen energetic neutral atoms (ENAs) originating in the Martian magnetospheath.

${ }^{\mathrm{b}}$ Hydrogen ENAs originating in undisturbed solar wind.

versus nadir direction can move to different angles and planet curvature is also taken into account.

[10] The altitude distributions of the main neutral species, $\mathrm{CO}_{2}, \mathrm{~N}_{2}, \mathrm{O}, \mathrm{H}_{2}$, and $\mathrm{He}$, were adopted from [Fox and Hac, 2009] for a low level of solar activity corresponding to the ASPERA-3 observations. The altitude profiles of the main neutral species adopted in the model are shown in Figure 1. Efficiency of the collisional thermalization and energy deposition of high-energy protons is determined by the elastic, ionization, and charge transfer collisions. If we suggest that

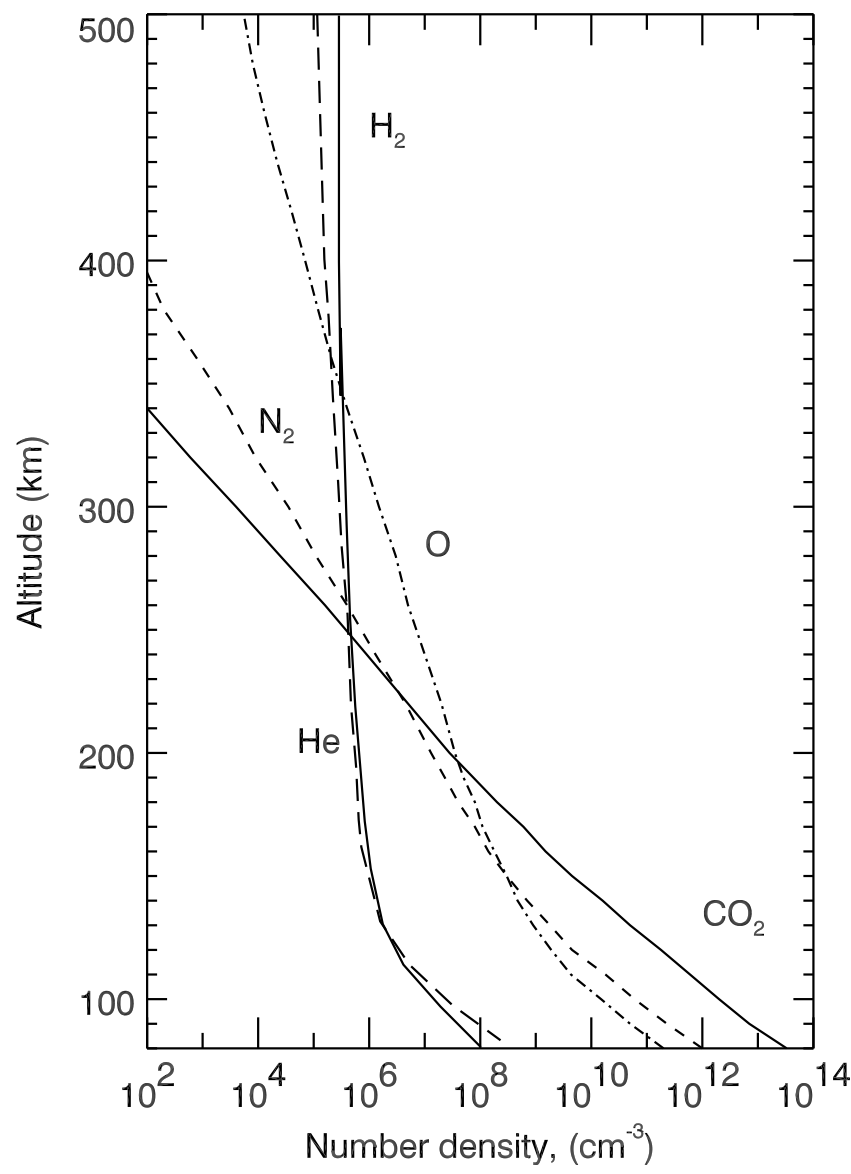

Figure 1. The altitude distributions of the main neutral species, $\mathrm{CO}_{2}, \mathrm{~N}_{2}, \mathrm{O}, \mathrm{H}_{2}$, and $\mathrm{He}$, adopted from Fox and Hac [2009]. characteristic value of total cross sections for collisions of all neutral species with protons at energy $1 \mathrm{keV}$ is about value of $10^{-15} \mathrm{~cm}^{2}$ (see, for example, Figure 2 for $\mathrm{H}^{+}$collisions with $\mathrm{CO}_{2}$ ) then the collisional frequencies below exobase will be proportional to the number densities of neutral species under consideration. From Figure 1 it is seen that at altitudes below
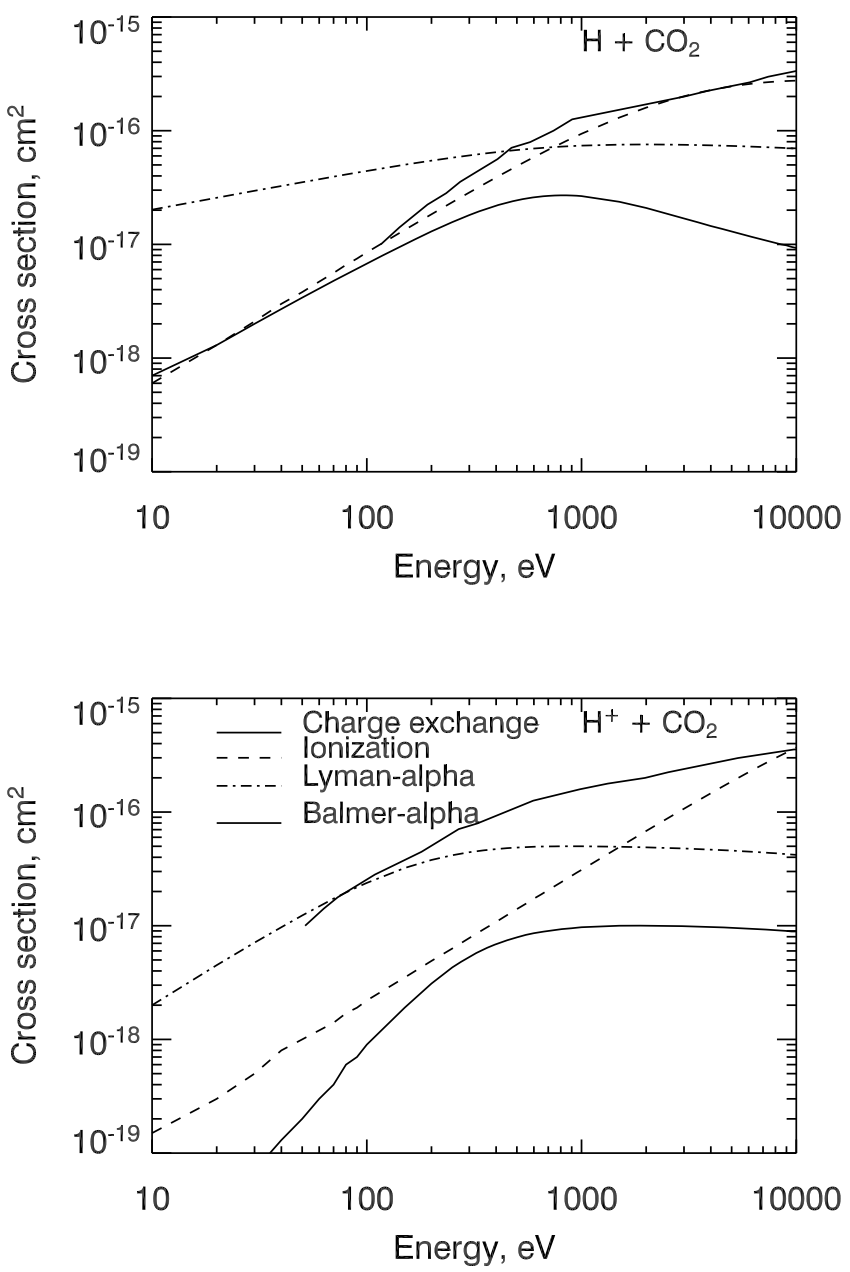

Figure 2. Cross sections for (top) $\mathrm{H}$ and (bottom) $\mathrm{H}^{+}$collisions with $\mathrm{CO}_{2}$. Processes are marked in the legend of Figure 2 (bottom). The solid line in Figure 2 (top) shows the stripping process. 


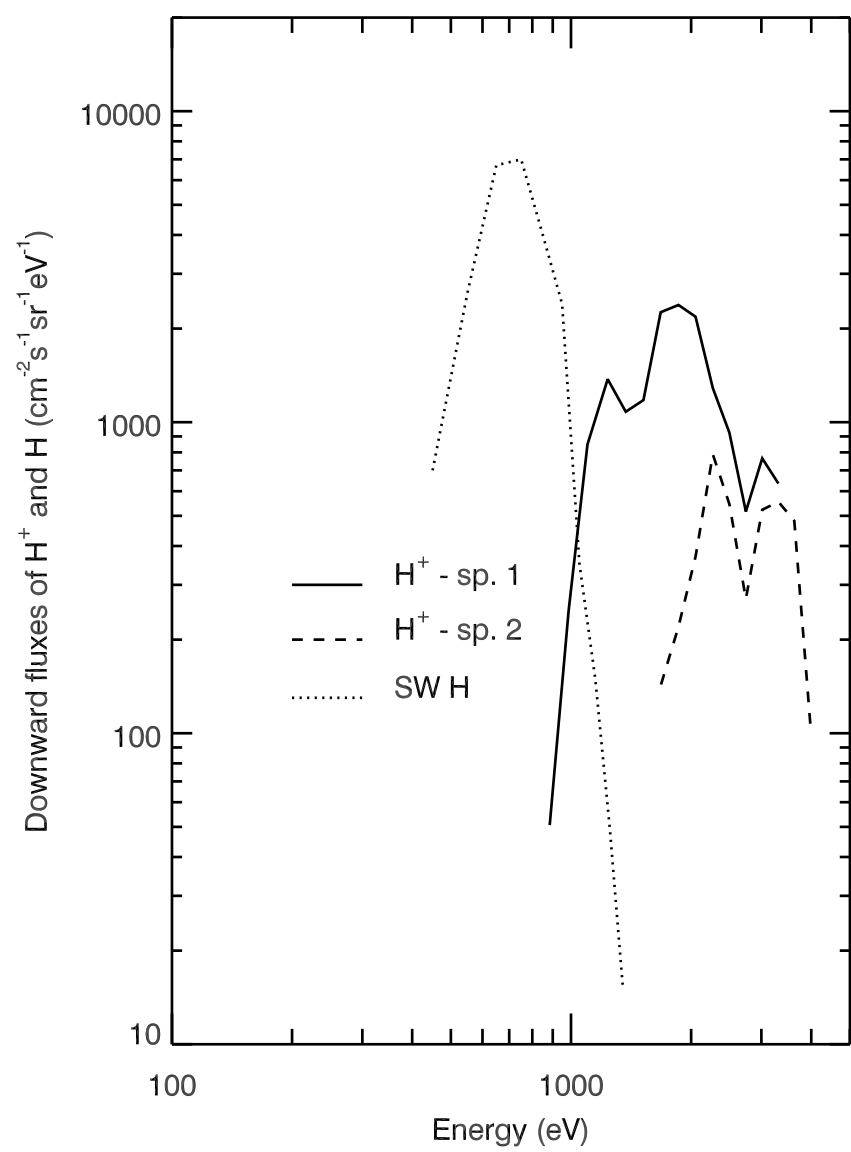

Figure 3. Energy spectra of the downward moving protons $\left(\mathrm{H}^{+}\right)$measured by ASPERA-3 and solar wind hydrogen atoms (SW H) calculated by Kallio et al. [1997], used as an input to the model at the upper boundary.

$200 \mathrm{~km}$ the number densities of light species $\mathrm{H}_{2}$ and $\mathrm{He}$ are more than 2 orders of magnitude lower then ones of heavy particles, therefore collisions with $\mathrm{CO}_{2}, \mathrm{~N}_{2}$, and $\mathrm{O}$ only were taken into account in the current version of the model aimed to calculate the proton energy deposition in the Martian upper atmosphere. Moreover, the cross sections of elastic collisions between protons and atmospheric species decrease with the proton energy, and are lower for the collisions with light $\left(\mathrm{H}_{2}\right.$ and $\left.\mathrm{He}\right)$ particles. The temperature in the considered domain changes from $150 \mathrm{~K}$ at lower boundary up to $170 \mathrm{~K}$ at the upper boundary.

[11] In the model, the most recent measurements or calculations of the required cross sections were adopted. The cross sections and scattering angle distributions for $\mathrm{H} / \mathrm{H}^{+}$ interactions with $\mathrm{N}_{2}$ and $\mathrm{O}$ are from the model of the proton aurora at Earth [Gérard et al., 2000]. The cross sections and scattering angle distributions for $\mathrm{H} / \mathrm{H}^{+}$collisions with $\mathrm{CO}_{2}$ are taken from Nakai et al. [1987] for charge exchange and stripping collisions, from Haider et al. [2002] for ionization, Lyman alpha and Balmer alpha excitation, and from Lindsay et al. [2005] for scattering angle distributions. The elastic and other inelastic collisions cross sections for $\mathrm{H} / \mathrm{H}^{+}$collisions with $\mathrm{CO}_{2}$ are assumed to be the same as for $\mathrm{O}_{2}$. The compiled cross sections for $\mathrm{H} / \mathrm{H}^{+}$collisions with $\mathrm{CO}_{2}$ are shown in Figure 2.
[12] The Monte Carlo code to model the penetration of high-energy protons and hydrogen atoms into the planetary atmosphere [Gérard et al., 2000] was modified to take into account the effect of the horizontal magnetic field on the proton trajectories. The induced magnetic field has been measured at Mars by the Mars Global Surveyor orbiter [Brain et al., 2003] and the Mars Express orbiter [Akalin et al., 2010]. These authors show that the induced magnetic field is mainly horizontal; its strength decreases with increasing altitude and with increasing solar zenith angle. The induced magnetic field strength is typically the strongest near the subsolar point (about $40 \mathrm{nT}$ ) and reaches a value of $20 \mathrm{nT}$ at the terminator, in the altitude range $360-440 \mathrm{~km}$ [Akalin et al., 2010]. Following these measurements we assume the constant in time and uniform in space horizontal magnetic field $\mathrm{B}=10,20,30,40$, and $50 \mathrm{nT}$ for the different runs.

\section{Results of Calculations}

[13] The most interesting effects of $\mathrm{H} / \mathrm{H}^{+}$penetration into the Mars upper atmosphere to investigate are heating of the neutral gas due to momentum transfer collisions, excitation of the precipitating hydrogen atoms and ambient gas emissions, and formation of the backscattered flux of the energetic hydrogen atoms and protons.

[14] The photon emissions and particle backscattered fluxes can be observed by the Spectroscopy for the Investigation of the Characteristics of the Atmosphere of Mars (SPICAM) and ASPERA-3 instruments orbiting Mars onboard the Mars Express mission. In this work, we use a spectrum of the downward moving protons, measured by the Mars Express ASPERA-3 [Barabash et al., 2006] in the energy range $700 \mathrm{eV}$ to $20 \mathrm{keV}$, and in altitude range $355-437 \mathrm{~km}$ at solar zenith angles $42^{\circ}-49^{\circ}$ on 27 February 2004, at around 19:35 UT. For this particular operational mode $700 \mathrm{eV}$ was the lower energy threshold. For this case, referred to as spectrum 1 (see Figure 3, solid line), the particle and energy fluxes of the incident protons were equal to $3.0 \times 10^{6} \mathrm{~cm}^{-2} \mathrm{~s}^{-1}$ and $1.4 \times 10^{-2} \mathrm{erg} \mathrm{cm}^{-2} \mathrm{~s}^{-1}$. To study the dependence on the input spectrum we also use another spectrum of downward moving protons measured by ASPERA-3, referred to as spectrum 2 (see Figure 3, dashed line). Spectrum 2 was measured in altitude range $387-435 \mathrm{~km}$ at solar zenith angles $30^{\circ}-33^{\circ}$ on 4 October 2005 at around 16:06 UT. Spectrum 2 is narrower in energy and the corresponding incident fluxes are of the same order of magnitude as those of spectrum 1 . A case study analysis of these proton spectra was reported by C. Diéval et al. (A case study of proton precipitation at Mars: Mars Express observations and hybrid simulation, submitted to Journal of Geophysical Research, 2011). It is necessary to point out that these proton spectra have been recorded when Mars Express spacecraft was in the ionosphere, thus below the IMB; that is, they are not magnetosheath spectra. The shapes of both spectra used in the calculations are typical as it follows from the statistical analysis of all proton precipitation events observed by Mars Express when the spacecraft was below the IMB, over a period of 1.5 years (C. Diéval, private communication, 2011). The proton events are rare: they are measured out of $1 \%$ of the observation time. The ASPERA ion instrument performs a full angular 


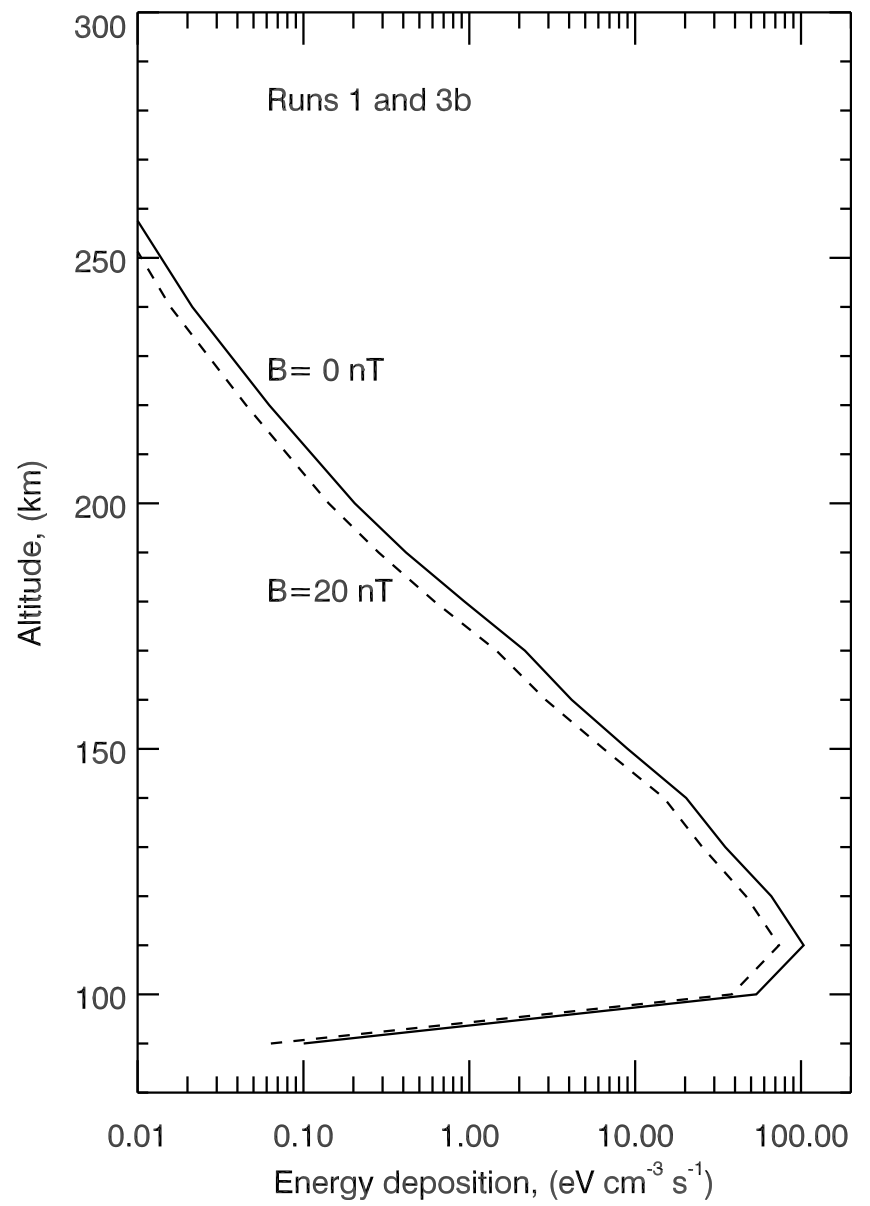

Figure 4. Energy deposition rates of precipitating protons for run without magnetic field (run 1 from Table 1) (solid line) and for run where $\mathrm{B}=20 \mathrm{nT}$ (run $3 \mathrm{~b}$ from Table 1) (dashed line).

scan in $192 \mathrm{~s}, 16$ angular steps. The proton flux inside the IMB is usually observed for shorter periods than this. We can therefore not obtain the full distribution. Assuming it to be isotropic is then a reasonable compromise, and we did try other distributions as well.

[15] We have conducted a set of runs to estimate the magnitudes of the $\mathrm{H} / \mathrm{H}^{+}$backscattered fluxes from the Martian atmosphere. The model input parameters are: energy spectrum and angle distribution versus nadir of the incident protons (or hydrogen atoms); magnitude of the parallel (horizontal to the surface of Mars) component of the induced magnetic field. The angle $\theta$ distribution is isotropic in the sense of the uniform distribution of $\cos \theta$. Such isotropic distribution is routinely used to study the proton precipitation in the Earth's polar atmosphere [Decker et al., 1996; Gérard et al., 2000]. We have conducted 8 runs, 6 for proton and 2 for hydrogen precipitation, which are listed in Table 1 . The upper boundary was taken at altitudes $355 \mathrm{~km}$ and $437 \mathrm{~km}$ for runs $3 \mathrm{a}$ and $3 \mathrm{~b}$ with spectrum 1 , and $-387 \mathrm{~km}$ and $435 \mathrm{~km}$ for runs $6 \mathrm{a}$ and $6 \mathrm{~b}$ with spectrum 2 . For all other runs $1-6$ the upper boundary was assigned at $437 \mathrm{~km}$ just to exclude the influence of the neutral Martian corona on the precipitating flux of protons.

\subsection{Run Without Induced Magnetic Field}

[16] To validate the developed DSMC model and to compare its outputs with previous studies [Kallio et al., 1997; Kallio and Barabash, 2001], we first have run the model for the case without an induced magnetic field in the Martian upper atmosphere. Figure 4 shows the energy deposition rate for the $\mathrm{H} / \mathrm{H}^{+}$flux penetrating through the Martian atmosphere. It is seen that in run 1 (without induced magnetic field) (Figure 4, solid line) the incident protons and hydrogen atoms penetrate rather deep into the thermosphere of Mars down to $100-150 \mathrm{~km}$ where the maximum of the energy deposition is reached.

[17] The calculated energy spectra of the protons and hydrogen atoms moving upward at the model upper boundary $h_{u b}=437 \mathrm{~km}$ are given in Figure 5. In the calculations with the DSMC model it was found that the interaction of the precipitating protons with the upper atmosphere results in the formation of an upward moving particle flux of $2.0 \times$ $10^{5} \mathrm{~cm}^{-2} \mathrm{~s}^{-1}$ and $7.0 \times 10^{5} \mathrm{~cm}^{-2} \mathrm{~s}^{-1}$ (protons and hydrogen atoms, respectively) and an energy flux of $5.7 \times 10^{-4} \mathrm{erg} \mathrm{cm}^{-2}$ $\mathrm{s}^{-1}$ and $1.3 \times 10^{-3} \mathrm{erg} \mathrm{cm}^{-2} \mathrm{~s}^{-1}$ (protons and hydrogen atoms, respectively) at the altitude $437 \mathrm{~km}$. In the "nonmagnetic" case $22 \%$ of the particle flux and $12 \%$ of the energy flux of the precipitating protons are backscattered as upward moving protons and hydrogen atoms by the Martian upper atmosphere. The energy spectrum of the up going protons follows

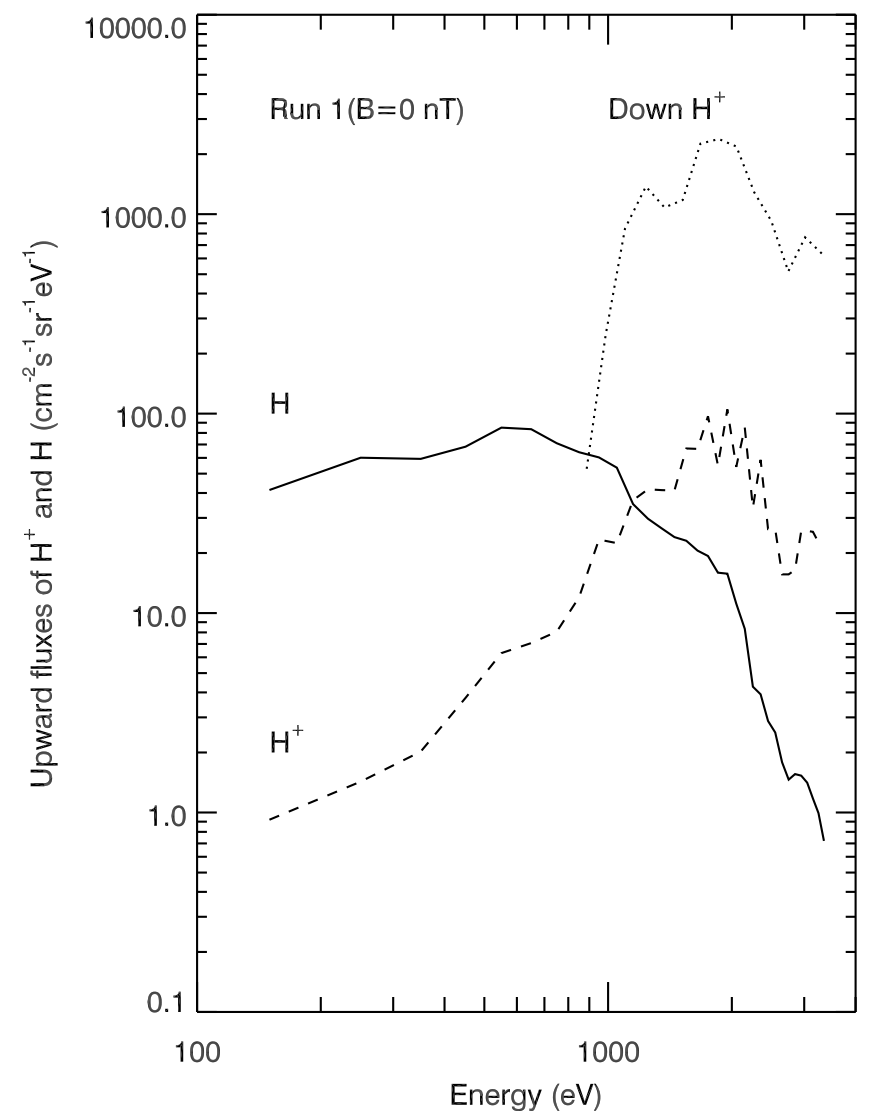

Figure 5. Energy spectra of the downward (spectrum 1 marked by dotted line) and upward (dashed line) moving protons and upward moving hydrogen atoms (solid line) for the run without induced magnetic field. 
a)

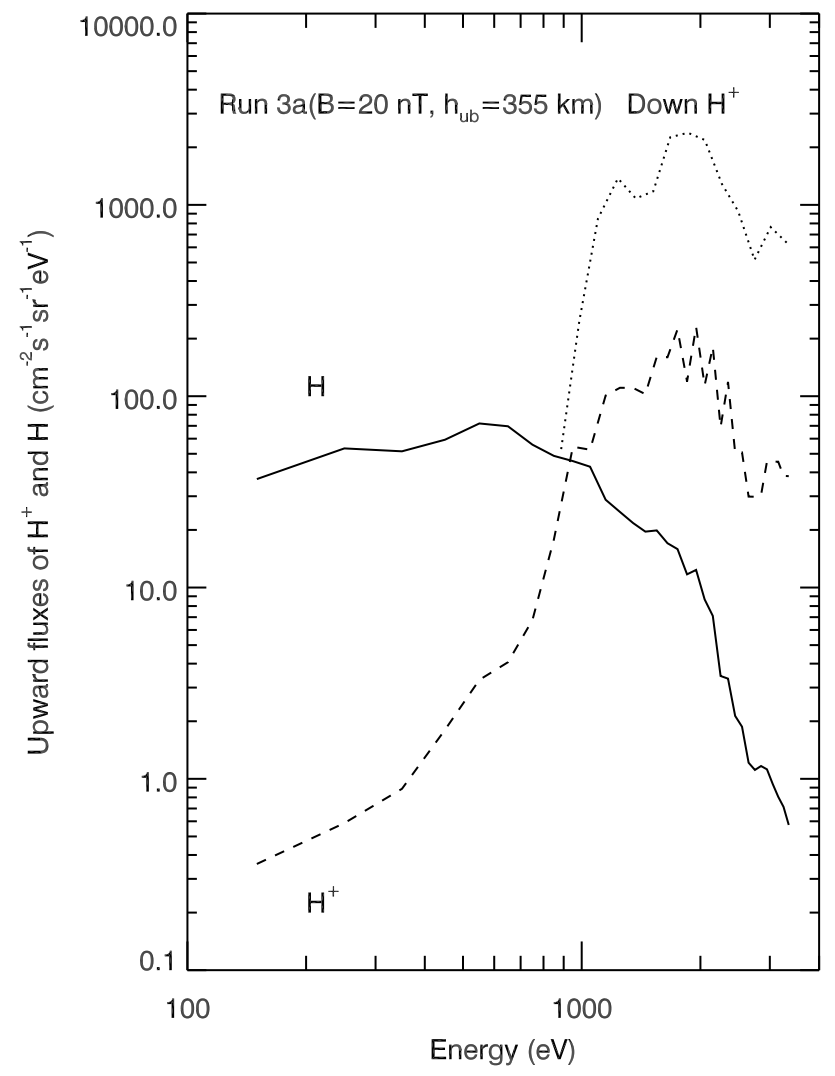

b)

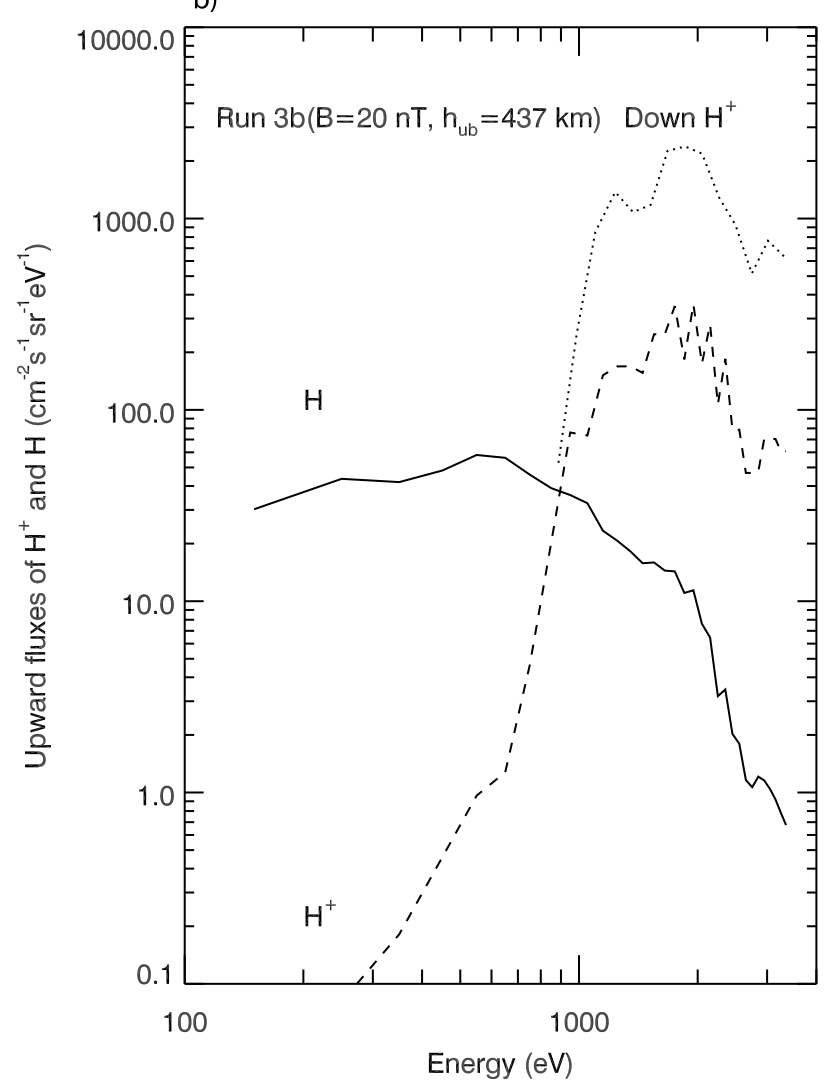

Figure 6. (a) Energy spectra of the downward (spectrum 1 at $h_{\mathrm{ub}}=355 \mathrm{~km}$ marked by dotted line) and upward (dashed line) moving protons and upward moving hydrogen atoms (solid line) for the run with the $20 \mathrm{nT}$ horizontal component of the induced magnetic field. (b) Energy spectra of the downward (spectrum 1 at $\mathrm{h}_{\mathrm{ub}}=437 \mathrm{~km}$ marked by dotted line) and upward (dashed line) moving protons and upward moving hydrogen atoms (solid line) for the run with the $20 \mathrm{nT}$ horizontal component of the induced magnetic field.

well the spectrum of the precipitating protons due to the single backward scattering at large angles (reflection) in the nadir direction which almost conserves energy due to large difference in masses for protons and atmospheric gas components. The low-energy part of spectrum $(<700 \mathrm{eV})$ is formed by the collisional spreading in the thermosphere due to multiple collisions. The spectrum of the up moving hydrogen atoms has a significantly stronger low-energy component also formed by collisional spreading due to multiple collisions in the altitude range of the maximum energy deposition rate (Figure 4, solid line).

[18] These estimates of the backscattered energy flux of the precipitating protons are lower than the values calculated by Kallio and Barabash [2001] for the precipitation of highenergy hydrogen atoms into the Martian upper atmosphere. Kallio and Barabash [2001] found that the up going hydrogen particle and energy fluxes constitute $58 \%$ and $33 \%$ of the impinging particle and energy fluxes, respectively. The difference could be explained by the fact that in the present model we use the updated set of cross sections and scattering angle distributions for each collisional process instead of the hard sphere approximation used in the previous models. It is well known that the hard sphere approximation based on the isotropic scattering angle distribution results in a very high rate of collisional spreading of the penetrating flux of protons and hydrogen atoms in a planetary atmosphere [Gérard et al., 2000, 2005].

\subsection{Runs With the Induced Magnetic Field}

[19] For the incident proton spectrum 1 we conducted two runs $3 \mathrm{a}$ and $3 \mathrm{~b}$ with the most probable value of the horizontal component $\mathrm{B}=20 \mathrm{nT}$ of the induced magnetic field. In these runs the model upper boundary was changed in accordance with the altitude range of the spectrum 1 measurements by ASPERA-3 instrument; that is, $h_{u b}$ was taken equal to $355 \mathrm{~km}$ in run $3 \mathrm{a}$, and $h_{u b}$ was taken equal to $437 \mathrm{~km}$ in run $3 \mathrm{~b}$. Figures $6 \mathrm{a}$ and $6 \mathrm{~b}$ show the energy spectra of the incident and backscattered protons and upward moving hydrogen atoms at the altitudes $h_{u b}=355 \mathrm{~km}$ (run 3a) and altitudes $h_{u b}=437 \mathrm{~km}$ (run $3 \mathrm{~b}$ ). It is necessary to point out that the value of $82 \mathrm{~km}$ altitude range during the measurement of spectrum 1 by the ASPERA-3 instrument is approaching the value of gyroradius for the precipitating protons ( $\sim 125 \mathrm{~km}$ for $2 \mathrm{keV}$ protons for conditions of run 3 ). Therefore, precipitating protons in run $3 \mathrm{a}$ have a higher probability to reach a collision-dominated region and deposit practically all their kinetic energy in this region. We have obtained the following estimates of the relative backscattered (upward) energy fluxes (EBF) for protons and hydrogen atoms: $\mathrm{EBF}_{\mathrm{p}}=16$, and $30 \%$ and $\mathrm{EBF}_{\mathrm{H}}=7$, and 
Table 2. Calculated Values of the Backscattered Energy Fluxes of $\mathrm{H} / \mathrm{H}^{+}$

\begin{tabular}{|c|c|c|c|c|c|c|}
\hline Run & B, Horizontal (nT) & Precipitating Spectra, $\mathrm{H}^{+}$and $\mathrm{H}$ at Altitude $\mathrm{h}_{\mathrm{ub}}$ & $\begin{array}{c}\text { Energy Up, } \\
\text { H }(\%)\end{array}$ & $\begin{array}{c}\text { Energy Up, } \\
\mathrm{H}^{+}(\%)\end{array}$ & $\begin{array}{l}\text { Energy Up, } \\
\text { Total (\%) }\end{array}$ & $\begin{array}{c}\mathrm{H} / \mathrm{H}^{+} \text {Flux Up, } \\
\text { Total }(\%)\end{array}$ \\
\hline 1 & 0 & Spectrum 1 , none, $\mathrm{h}_{\mathrm{ub}}=437 \mathrm{~km}$ & 8 & 4 & 12 & 22 \\
\hline 2 & 10 & Spectrum 1 , none, $\mathrm{h}_{\mathrm{ub}}=437 \mathrm{~km}$ & 7 & 11 & 18 & 27 \\
\hline $3 a$ & 20 & Spectrum 1 , none, $\mathrm{h}_{\mathrm{ub}}=355 \mathrm{~km}$ & 7 & 16 & 23 & 33 \\
\hline $3 b$ & 20 & Spectrum 1, none, $\mathrm{h}_{\mathrm{ub}}=437 \mathrm{~km}$ & 6 & 30 & 36 & 45 \\
\hline 4 & 30 & Spectrum 1 , none, $\mathrm{h}_{\mathrm{ub}}=437 \mathrm{~km}$ & 5 & 53 & 58 & 66 \\
\hline 5 & 50 & Spectrum 1, none, $\mathrm{h}_{\mathrm{ub}}=437 \mathrm{~km}$ & 3 & 89 & 92 & 95 \\
\hline $6 \mathrm{a}$ & 20 & Spectrum 2, none, $\mathrm{h}_{\mathrm{ub}}=387 \mathrm{~km}$ & 4 & 16 & 20 & 26 \\
\hline $6 \mathrm{~b}$ & 20 & Spectrum 2, none, $\mathrm{h}_{\mathrm{ub}}=435 \mathrm{~km}$ & 4 & 24 & 28 & 34 \\
\hline 7 & 20 & None, MS H, $\mathrm{h}_{\mathrm{ub}}=500 \mathrm{~km}$ & 12 & 0.3 & 12 & 19 \\
\hline 8 & 20 & None, $\mathrm{SW} \mathrm{H}, \mathrm{h}_{\mathrm{ub}}=500 \mathrm{~km}$ & 10 & 0.3 & 10 & 15 \\
\hline
\end{tabular}

$6 \%$ for runs $3 \mathrm{a}$ and $3 \mathrm{~b}$ with the horizontal magnetic field $\mathrm{B}=20$, correspondingly (see Table 2 ). It is seen that a value of $\mathrm{EBF}_{\mathrm{p}}$ strongly depends on the selected value of the model upper boundary $h_{u b}$, but $\mathrm{EBF}_{\mathrm{H}}$ practically does not change in runs $3 \mathrm{a}$ and $3 \mathrm{~b}$. Therefore, to exclude the direct influence of the neutral atmosphere on the flux of precipitating protons we used the value $h_{u b}=437 \mathrm{~km}$ as an upper boundary for our model.

[20] For the incident proton spectrum 1 and for the different magnitudes of the horizontal component of the induced magnetic field $\mathrm{B}$ we have obtained the following estimates of the relative backscattered (upward) energy fluxes for protons and hydrogen atoms: $\mathrm{EBF}_{\mathrm{p}}=11 \%, 30 \%$, $53 \%$, and $89 \%$ and $\mathrm{EBF}_{\mathrm{H}}=7 \%, 6 \%, 5 \%$, and $3 \%$ for the horizontal magnetic field $\mathrm{B}=10,20,30$, and $50 \mathrm{nT}$, correspondingly (see Table 2).

[21] The shapes of the upward spectra are similar to the case without magnetic field except the low-energy proton component is gone but the absolute fluxes are substantially higher. For the measured spectrum of the incident protons and the relatively high magnitude of the horizontal (parallel) magnetic field $\mathrm{B}=50 \mathrm{nT}$ the thermosphere of Mars is practically shielded from proton precipitation (the up going flux reaches $95 \%$ of the down going flux). Indeed, in this case the gyroradius for precipitating protons $(\sim 125 \mathrm{~km}$ for $2 \mathrm{keV}$ protons) is smaller than the distance between the top boundary and the collision dominated region $(420 \mathrm{~km})$. Therefore, all protons return back even before they reach altitudes where collisions start playing role. The up going neutral flux is due to small amount of high-energy protons which managed penetrate through the magnetic field. The cases with moderate magnitudes of the horizontal magnetic field result in values of the backscattered fluxes of protons and hydrogen atoms that are comparable with the previous estimates based on the ASPERA-3 measurements. The dependence of the upward flux energy spectra on the value of the horizontal magnetic field is presented in Figure 7.

[22] The presence of the magnetic field changes not only the upward flux but also the energy deposition rate and the excitation of gas emissions. Figure 4 shows the changes in the energy deposition rate due to the presence of the magnetic field. For the case with the magnetic field $\mathrm{B}=20 \mathrm{nT}$ the heating of the neutral gas due to momentum transfer collisions decreases ( $37 \%$ at the peak) due to significant increase of the proton albedo.

[23] We have also conducted two sensitivity runs. Two runs were conducted for the reference case $3 \mathrm{~b}$ but the cross sections of the $\mathrm{CO}_{2}$ collisions with protons and hydrogen atoms were enlarged and decreased by a factor of 10 . In the reference case the elastic and inelastic cross sections for $\mathrm{CO}_{2}$ collisions with $\mathrm{H} / \mathrm{H}^{+}$are taken to be the same as for $\mathrm{O}_{2}$ molecule and are thus uncertain. The following values of the upward energy flux of $\mathrm{EBF}_{\mathrm{H}}=13 \%$ cross sections (CSs) enlarged by 10$)$, and $3 \%(\mathrm{CSs}$ decreased by 10$)$ and $\mathrm{EBF}_{\mathrm{P}}=$ $30 \%$ and $30 \%$ were obtained. It is seen that difference with the reference run $3 \mathrm{~b}$ is about a factor of 2 for the value of $\mathrm{EBF}_{\mathrm{H}}$ and is practically negligible for $\mathrm{EBF}_{\mathrm{p}}$. In the other sensitivity run the dependence on the angle distribution of the

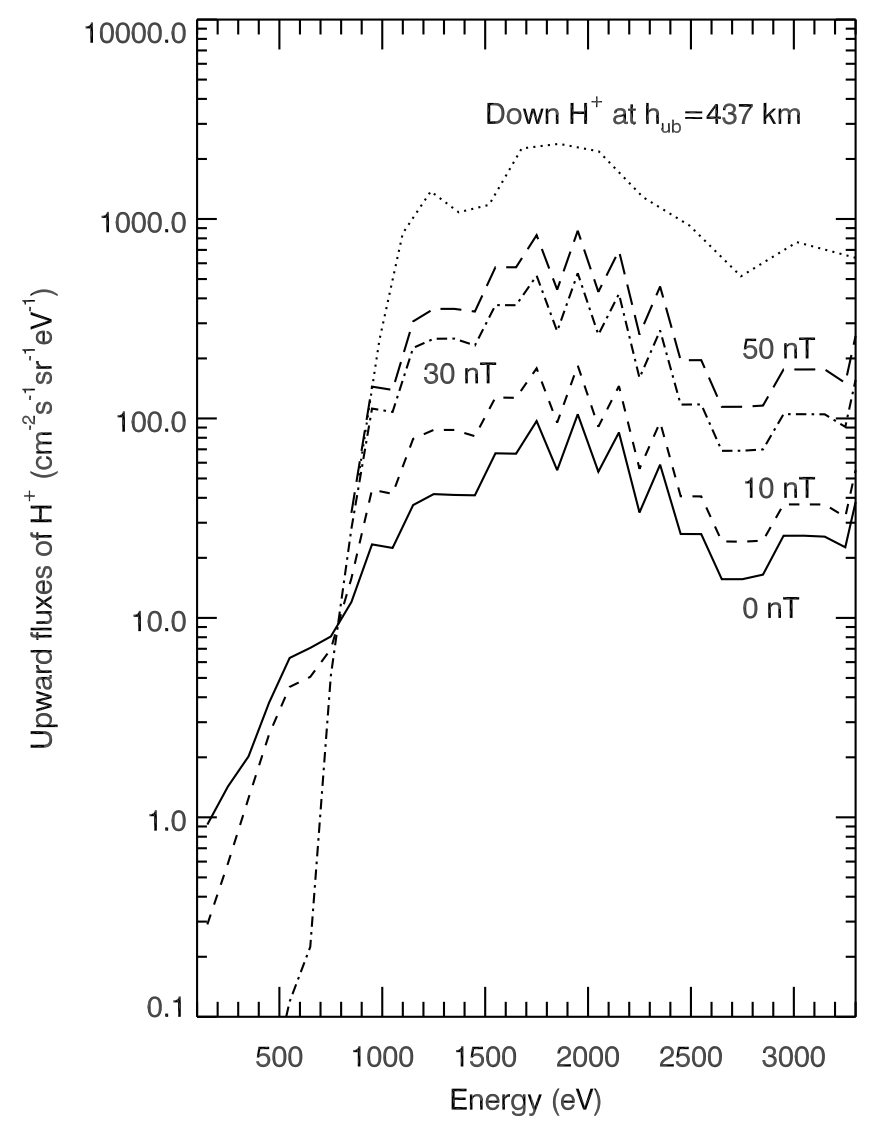

Figure 7. Energy spectra of the upward proton fluxes for different values of the horizontal magnetic field B. Solid line denotes the run for $\mathrm{B}=0$, short-dashed line denotes the run for $\mathrm{B}=10$, dashed-dotted line denotes the run for $\mathrm{B}=30$, long-dashed line denotes the run for $\mathrm{B}=50$, and the dotted line shows the downward spectrum 1 . 
a)

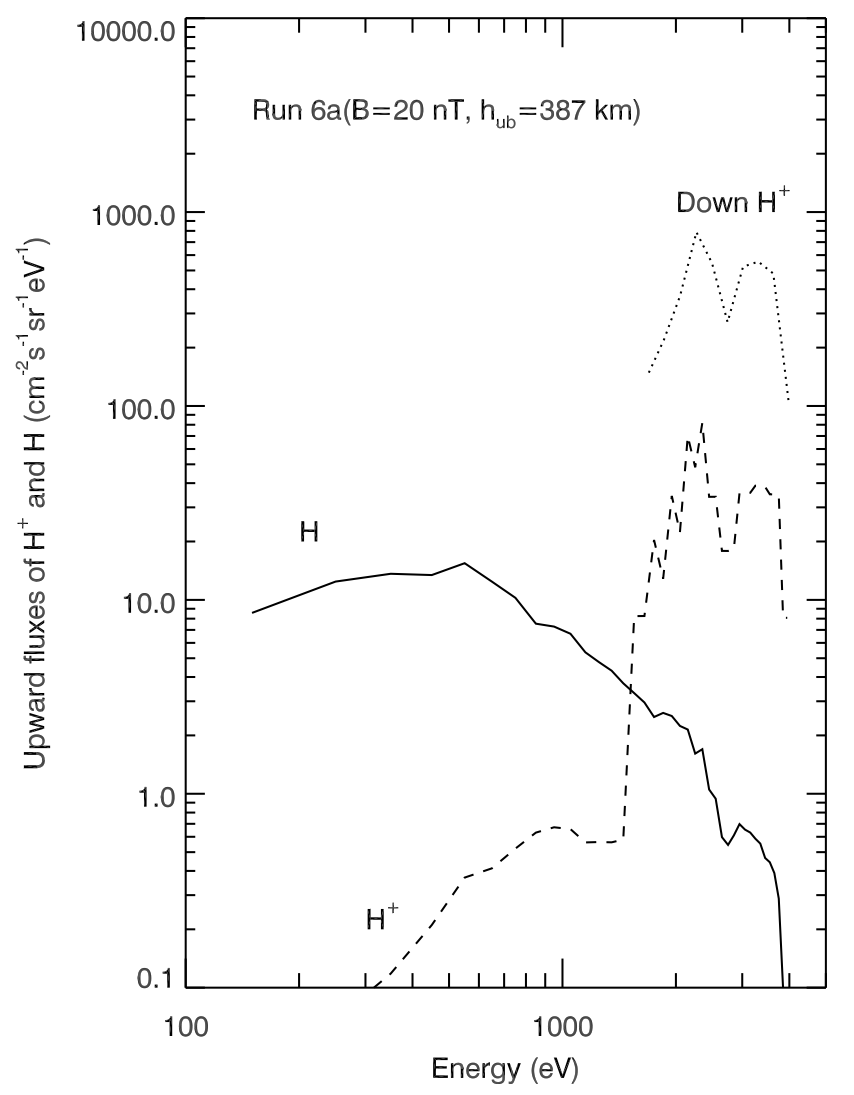

b)

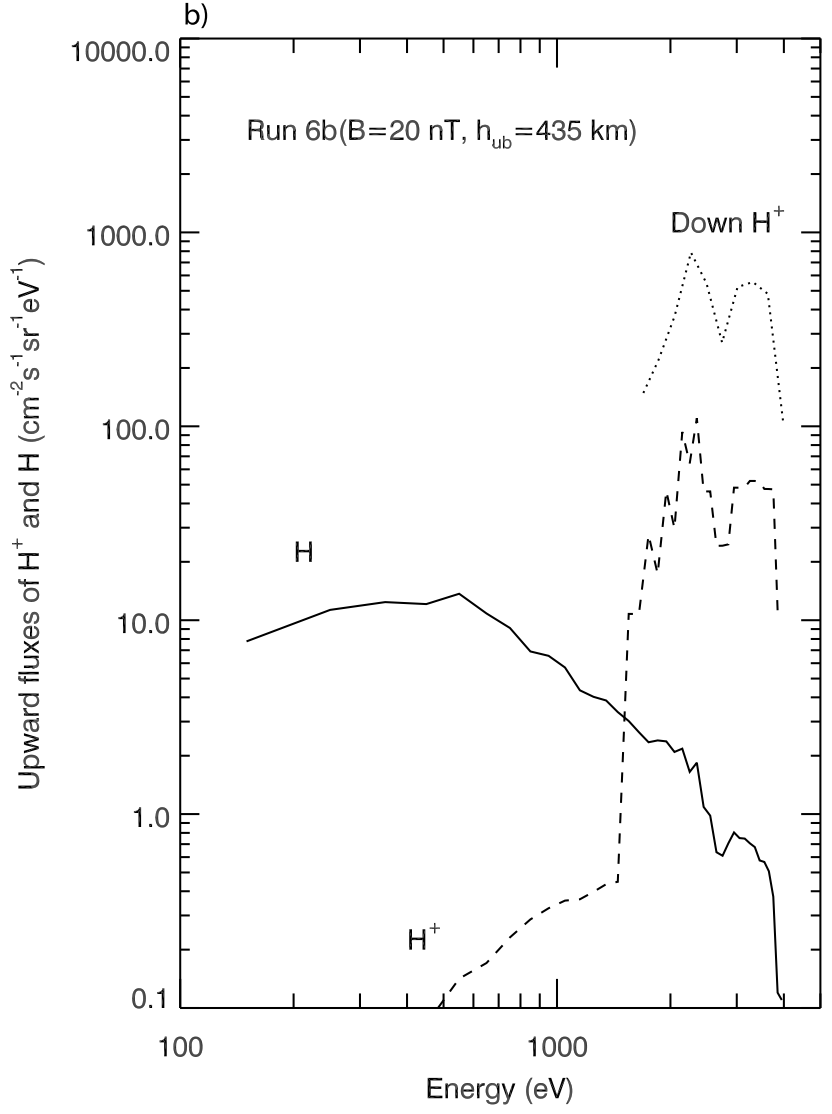

Figure 8. (a) Energy spectra of the downward (spectrum 2 at $h_{u b}=387 \mathrm{~km}$ marked by dotted line) and upward (dashed line) moving protons and upward moving hydrogen atoms (solid line) for the run with the $20 \mathrm{nT}$ horizontal component of the induced magnetic field. (b) Energy spectra of the downward (spectrum 2 at $\mathrm{h}_{\mathrm{ub}}=435 \mathrm{~km}$ marked by dotted line) and upward (dashed line) moving protons and upward moving hydrogen atoms (solid line) for the run with the $20 \mathrm{nT}$ horizontal component of the induced magnetic field.

incident protons was investigated. In this run a nonisotropic distribution (the angle to the nadir direction was limited to the range $\pm 45^{\circ}$ ) for the precipitating flux of protons was considered. The upward energy flux was for $\mathrm{H}=6 \%$, and $\mathrm{P}=$ $25.5 \%$. Again, it was found that the difference is not large. There is a small reduction for protons due to additional collisions, but the backscattered fluxes of protons and hydrogen atoms due to gyromotion are practically the same. This is caused by the deeper penetration of the $\mathrm{H} / \mathrm{H}^{+}$flux into the Martian thermosphere and, as a consequence, a more efficient collisional thermalization of the incident particles.

[24] For the incident proton spectrum 2 we also conducted two runs $6 \mathrm{a}$ and $6 \mathrm{~b}$ with the most probable value of the horizontal component $\mathrm{B}=20 \mathrm{nT}$ of the induced magnetic field. In these runs the model upper boundary was changed in accordance with the altitude range of the spectrum 2 measurements by ASPERA-3 instrument; that is, $h_{u b}$ was taken equal to $387 \mathrm{~km}$ in run $6 \mathrm{a}$, and $h_{u b}$ was taken equal to $435 \mathrm{~km}$ in run $6 \mathrm{~b}$. Figures $8 \mathrm{a}$ and $8 \mathrm{~b}$ show the energy spectra of the incident and backscattered protons and upward moving hydrogen atoms at the altitudes $h_{u b}=387 \mathrm{~km}$ (run 6a) and altitudes $h_{u b}=435 \mathrm{~km}$ (run 6b). For the incident proton spectrum 2 (run 6 with $\mathrm{B}=20 \mathrm{nT}$ ) the upward fluxes for protons and hydrogen are $\mathrm{EBF}_{\mathrm{H}}=4 \%$, and $4 \%$ and $\mathrm{EBF}_{\mathrm{P}}=$
$16 \%$, and $24 \%$ for runs $6 \mathrm{a}$ and $6 \mathrm{~b}$, correspondingly. The relative upward energy fluxes were $20 \%$ and $28 \%$, and upward particle fluxes $34 \%$ and $40 \%$ for runs $6 \mathrm{a}$ and $6 \mathrm{~b}$. The calculated energy spectra of the protons and hydrogen atoms moving upward at the model upper boundary are shown in Figure 8. Comparison of Figures 6 and 8 shows that the overall pattern qualitatively remains the same. The mean energy of the spectrum 2 is higher (see Figure 3), therefore the low-energy upward proton flux is more developed, and the hydrogen up going flux is shifted toward lower energies because of collisional spreading in the altitude range of maximum energy deposition.

[25] The Martian upper atmosphere is under the influence of an intense flux of hydrogen atoms of the solar wind energy [Kallio and Barabash, 2001]. In the solar wind and in the magnetosheath, hydrogen ENAs are produced in the charge exchange between solar wind protons and the hydrogen corona either beyond the bow shock (undisturbed solar wind) or in the magnetosheath. Two runs were conducted for fluxes of hydrogen ENAs precipitating onto the Martian atmosphere at a height of $500 \mathrm{~km}$. The respective spectra were taken from Kallio et al. [1997, Figure 10]. In run 7 the incident $H$ spectrum corresponds to the magnetosheath (MS) energetic neutral atoms (ENAs) and in run 8 it corresponds to the solar 


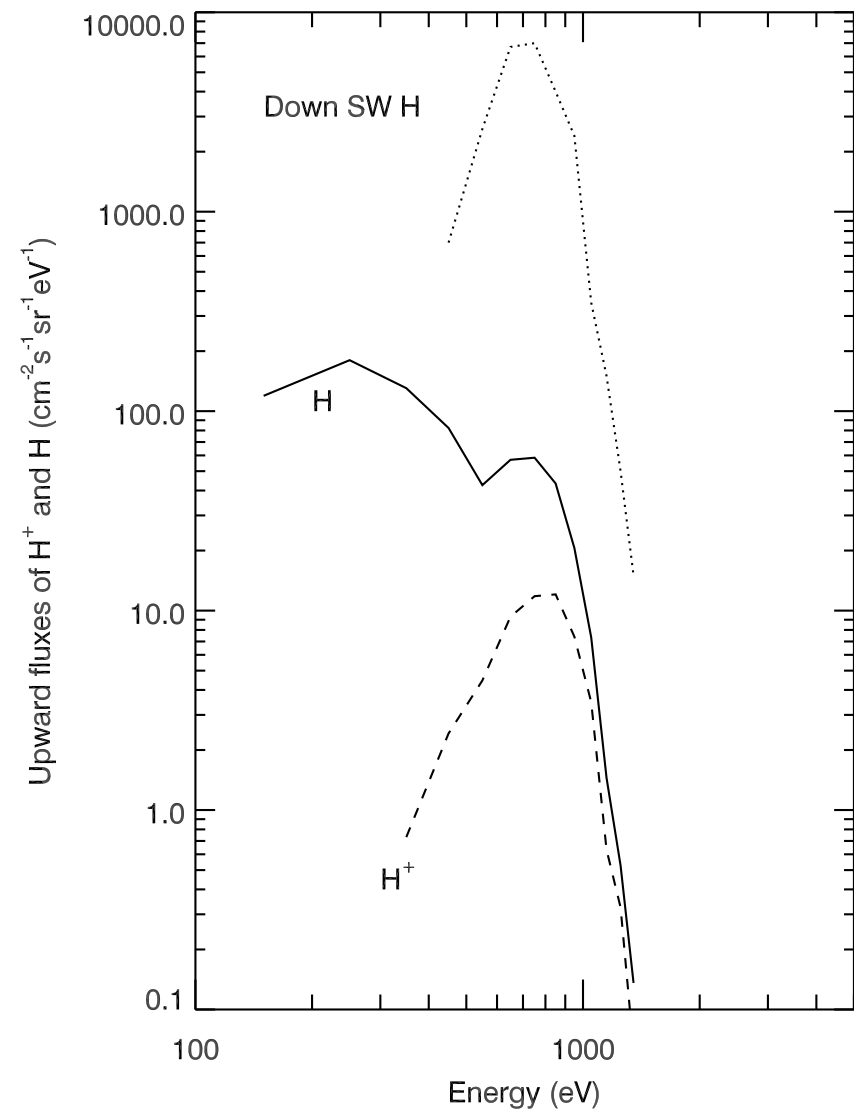

Figure 9. Energy spectra of the downward (dotted line) and upward (dashed line) moving hydrogen atoms and upward moving protons (solid line) for run 8 .

wind (SW) ENAs (Figure 3, dotted line). In run 7 the spectrum of MS H ENAs was approximated by a flat distribution with a value of $3 \times 10^{6} \mathrm{~cm}^{-2} \mathrm{~s}^{-1} \mathrm{eV}^{-1}$ for the energy interval from 100 to $900 \mathrm{eV}$. In run 7 the upward energy flux of hydrogen was $12 \%$, and protons $0.3 \%$. For run 8 the upward energy flux of hydrogen was $10 \%$, and of protons $0.3 \%$. The calculated energy spectra of the protons and hydrogen atoms moving upward at the model upper boundary are given in Figure 9. An additional run, identical to run 8 but without induced magnetic field, was conducted. For this run the upward energy fluxes of hydrogen atoms and protons were found to be practically the same as in run 8 . Again, the backscattered flux of $\mathrm{H}$ is formed mainly due to the collisional spreading in the Martian thermosphere, while the upward moving flux of protons is formed due to the stripping in the upper layers of the atmosphere and therefore it follows the energy spectrum of the incident hydrogen atoms at altitude $500 \mathrm{~km}$.

[26] The calculated estimates of the energy and particle fluxes of backscattered protons and hydrogen atoms obtained in runs 1-8 are summed in Table 2.

[27] The total amount of up going particles $\left(\mathrm{H}\right.$ and $\left.\mathrm{H}^{+}\right)$is about the same in runs 1,7 , and 8 . This indicates the down going flux reaches the exobase nonaffected either due to neutrality (run 7 and 8) or the absence of the magnetic field (run 1). Runs 7 and 8 show that very few protons leave the system. As it can be seen in Figure 2 (top) the cross section for stripping is comparable to the ionization cross section. This leads to the formation of protons in the collision dominated region, and as a consequence to the reduction of the backscattered flux.

\section{Summary and Conclusions}

[28] A DSMC model for the calculations of the transport of high-energy proton and hydrogen atoms in the Martian upper atmosphere has been developed. It takes into account all physical processes of the $\mathrm{H} / \mathrm{H}^{+}$penetration through the atmosphere. The important features of the elaborated model are: the detailed consideration of the scattering angle in each collision and utilization of the most recent set of cross sections.

[29] This code is used to calculate the backscattered flux of energetic hydrogen atoms and protons and the total energy deposition rates. The calculations have been performed for the Martian atmosphere during the solar minimum. We use a spectrum of the incident protons in the altitude range 355 $437 \mathrm{~km}$ measured by the Mars Express ASPERA-3 in the energy range $700 \mathrm{eV}$ to $20 \mathrm{keV}$. The particle and energy fluxes of the incident protons were equal to $3.0 \times 10^{6} \mathrm{~cm}^{-2} \mathrm{~s}^{-1}$ and $1.4 \times 10^{-2} \mathrm{erg} \mathrm{cm}^{-2} \mathrm{~s}^{-1}$. It was found that $22 \%$ of particle flux and $12 \%$ of the energy flux of the precipitating protons were backscattered by the Martian upper atmosphere if no magnetic field is taken into account.

[30] The horizontal magnetic field induced by solar wind leads to an increase of the $\mathrm{H}^{+}$upward flux because of the proton gyromotion. Including a $20 \mathrm{nT}$ horizontal magnetic field in the altitude range of $85-500 \mathrm{~km}$ in the model increases the backscattered up to $40 \%-50 \%$. The $50 \mathrm{nT}$ field almost completely screen off the precipitating flux. The magnetic field plays a crucial role in the transport of charged particles and determines the energy deposition of the solar wind protons in the upper atmosphere of Mars.

[31] Acknowledgments. The contribution by V.S. and D.B. was supported by the Swedish Research Council via grant "Physics of induced magnetospheres" to the Swedish Institute of Space Physics and the Basic Research Program of the Presidium of the Russian Academy of Sciences, Russian Foundation for Basic Research (project 11-02-00479a), and the Federal Targeted Program "Science and Science Education for Innovation in Russia 2009-2013." This study was also supported by the Belgium National Found for Scientific Research (FNRS). C.D. was supported by the National Graduate School of Space Technology of Luleå Technical University.

[32] Masaki Fujimoto thanks the reviewers for their assistance in evaluating this paper.

\section{References}

Akalin, F., D. D. Morgan, D. A. Gurnett, D. L. Kirchner, D. A. Brain R. Modolo, M. H. Acuna, and J. R. Espley (2010), Dayside induced magnetic field in the ionosphere of Mars, Icarus, 206, 104-111, doi:10.1016/j.icarus.2009.03.021

Barabash, S., et al. (2006), The analyzer of space plasmas and energetic atoms (ASPERA-3) for the Mars Express mission, Space Sci. Rev., 126(1-4), 113-164

Bisikalo, D. V., V. I. Shematovich, and J.-C. Gérard (1995), Kinetic model of the formation of the hot oxygen geocorona: 2. Influence of $\mathrm{O}^{+}$ion precipitation, J. Geophys. Res., 100, 3715-3720, doi:10.1029/94JA03196.

Brain, D. A., F. Bagenal, M. H. Acuna, and J. E. P. Connerney (2003), Martian magnetic morphology: Contributions from the solar wind and crust, J. Geophys. Res., 108(A12), 1424, doi:10.1029/2002JA009482.

Decker, D. T., B. V. Kozelov, B. Basu, J. R. Jasperse, and V. E. Ivanov (1996), Collisional degradation of the proton-H atom fluxes in the atmosphere: A comparison of theoretical techniques, J. Geophys. Res., 101, 26,947-26,960, doi:10.1029/96JA02679. 
Fox, J. L., and A. B. Hac (2009), Photochemical escape of oxygen from Mars: A comparison of the exobase approximation to a Monte Carlo method, Icarus, 204, 527-544, doi:10.1016/j.icarus.2009.07.005.

Futaana, Y., et al. (2006), First ENA observations at Mars: ENA emissions from the Martian upper atmosphere, Icarus, 182, 424-430, doi:10.1016/ j.icarus.2005.09.019.

Gérard, J.-C., B. Hubert, D. V. Bisikalo, and V. I. Shematovich (2000), A model of the Lyman- $\alpha$ line profile in the proton aurora, J. Geophys. Res. 105, 15,795-15,806, doi:10.1029/1999JA002002.

Gérard, J.-C., V. I. Shematovich, D. V. Bisikalo, and D. Lummerzheim (2005), A Monte Carlo model of auroral hydrogen emission line profiles, Ann. Geophys., 23, 1473-1480, doi:10.5194/angeo-23-1473-2005.

Haider, S. A., S. P. Seth, E. Kallio, and K. I. Oyama (2002), Solar EUV and electron-proton-hydrogen atom-produced ionosphere on Mars: Comparative studies of particle fluxes and ion production rates due to different processes, Icarus, 159, 18-30, doi:10.1006/icar.2002.6919.

Kallio, E., and S. Barabash (2001), Atmospheric effects of precipitating energetic hydrogen atoms on the Martian atmosphere, J. Geophys. Res., 106, 165-177, doi:10.1029/2000JA002003.

Kallio, E., J. G. Luhmann, and S. Barabash (1997), Charge exchange near Mars: The solar wind absorption and neutral atom production, J. Geophys. Res., 102, 22,183-22,197, doi:10.1029/97JA01662.

Lindsay, B. G., W. S. Yu, and R. F. Stebbings (2005), Cross sections for electron capture and loss by keV oxygen atoms in collisions with $\mathrm{CO}$ and $\mathrm{CO}_{2}, J$. Geophys. Res., 110, A02302, doi:10.1029/2004JA010854.

Lundin, R., et al. (2004), Solar wind-induced atmospheric erosion at Mars: First results from ASPERA-3 on Mars Express, Science, 305(5692), 1933-1936, doi:10.1126/science.1101860.
Moore, T. E., and J. L. Horwitz (2007), Stellar ablation of planetary atmospheres, Rev. Geophys., 45, RG3002, doi:10.1029/2005RG000194.

Nakai, Y., T. Shirai, T. Tabata, and R. Ito (1987), Cross sections for charge transfer of hydrogen atoms and ions colliding with gaseous atoms and molecules, At. Data Nucl. Data Tables, 37, 69-101, doi:10.1016/0092640X(87)90005-2.

Shematovich, V. I. (2008), Kinetics of suprathermal atoms and molecules in the rarefied planetary atmospheres, in Rarified Gas Dynamics, edited by T. Abe et al., AIP Conf. Proc., 1084, 1047-1054, doi:10.1063/ 1.3076436 .

Shematovich, V. I., D. V. Bisikalo, and J.-C. Gérard (1994), A kinetic model of the formation of the hot oxygen geocorona: 1. Quiet geomag netic conditions, J. Geophys. Res., 99, 23,217-23,228, doi:10.1029/ 94JA01769.

S. Barabash, C. Diéval, Y. Futaana, M. Holmstrom, H. Nilsson, and G. Stenberg, Swedish Institute of Space Physics, SE-98128 Kiruna, Sweden. (stas@irf.se; catherine@irf.se; futaana@irf.se; matsh@irf.se; hans.nilsson@irf.se; gabriella@irf.se)

D. V. Bisikalo and V. I. Shematovich, Institute of Astronomy, Russian Academy of Sciences, 48 Pyatnitskaya str., Moscow 119017, Russia (bisikalo@inasan.rssi.ru; shematov@inasan.ru)

J.-C. Gérard, LPAP, Université de Liège, B-4000 Liège, Belgium. (jc.gerard@ulg.ac.be) 